\title{
ASSOCIAÇÃO ENTRE OS NÍVEIS DE ATIVIDADE FÍSICA E DE ESTRESSE EM ESTUDANTES DO ENSINO MÉDIO DE TERESINA-PI
}

\author{
Márcia Cristiane Araújo \\ Centro Universitário Santo Agostinho - UNIFSA \\ Matheus José Acácio de Oliveira \\ Centro Universitário Santo Agostinho - UNIFSA \\ Ana Vitória Moreira Nogeura \\ Centro Universitário Santo Agostinho - UNIFSA \\ Francilene Batista Madeira \\ Universidade Estadual do Piauí - UESPI \\ Fernando Donatan Viegas Braga \\ Universidade do Norte do Paraná - UNOPAR \\ Herbert Gustavo Simões \\ Universidade Católica de Brasília - UCB \\ Nanci Maria de França \\ Universidade Católica de Brasília - UCB
}

\section{RESUMO}

Evidências epidemiológicas mostram que durante o ensino médio os adolescentes tendem a se tornarem menos ativos e mais estressados, devido ao aumento dos compromissos acadêmicos. o sedentarismo é um dos principais fatores de risco para o desenvolvimento de doenças crônicas degenerativas e transtornos psicossomáticos. Em contrapartida, o exercício físico é considerado uma das principais medidas não farmacológicas para o alívio do estresse e tem papel positivo sobre a saúde física e mental do indivíduo. Este estudo teve como objetivo estimar a prevalência do nível de atividade física (NAF) e do estresse em estudantes do 3ㅇaㅅ ano do ensino médio e verificar a associação entre as duas variáveis. $O$ estudo avaliou $\mathrm{n}=$ 141 adolescentes (idade $=17,6 \pm 0,4$ ), de uma escola particular da cidade de Teresina (PI), que estavam em fase de preparação para o exame nacional do ensino médio (ENEM). Foram empregados os instrumentos Questionário Internacional de Atividade Física (IPAQ-versão curta), para avaliar o NAF, e a Escala de Stress para Adolescentes (ESA), para avaliar o nível, a fase e a sintomatologia do estresse mais predominante nos estudantes. Foram empregados procedimentos estatísticos descritivos expressos por freqüência, percentual, média e desvio padrão. A associação entre as variáveis do NAF e de estresse da ESA foram analisadas por meio do Teste Qui-Quadrado. Os dados mostraram que $71,6 \%$ dos escolares se encontravam insuficientemente ativos e 70,2\% estavam estressados. Identificou-se a prevalência da fase de resistência nos adolescentes do sexo masculino e a fase de quase exaustão no sexo feminino, com predominância dos sintomas psicológicos para ambos os sexos. Houve associação significativa $(p=0,004)$ entre o fato do estudante se encontrar insuficientemente ativo e apresentar um nível de estresse prejudicial à saúde. Diante dos resultados encontrados, pode-se inferir que o sedentarismo pode potencializar os efeitos nocivos do estresse, durante essa etapa da vida e que a aproximação da data do ENEM contribuiu para aumentar os sintomas estressantes nessa amostra de estudantes. No entanto, sugere-se a realização de novas pesquisas envolvendo amostras maiores e com estudantes de escolas particulares e públicas, para verificar se os achados serão semelhantes aos encontrados neste estudo.

Palavras-Chave: Adolescentes. Sedentarismo. Estresse mental. 


\section{INTRODUÇÃO}

A adolescência é uma fase marcada por inúmeras mudanças biopsicossociais. Nessa etapa da vida, o indivíduo vivencia uma transição entre a fase infantil e a fase adulta, na qual exige grande esforço físico e mental para se adaptar, enfrentar uma nova realidade e para construir sua identidade (PATIAS; HEINE; DELL'AGLIO, 2017). Diversos autores consideram como uma das etapas da vida mais vulnerável ao estresse, haja vista que muitos adolescentes estão passando por: mudanças físicas, desequilíbrio hormonal, tipo e quantidade de responsabilidade, opção sexual, consumismo, novas tecnologias, conflito das drogas, relações interpessoais, escolha de uma carreira profissional (LIPP, MALAGRIS e NOVAIS, 2007; MARQUES; GASPAROTTO; COELHO, 2014).

É nesse contexto estressante da adolescência que o jovem se depara com a necessidade de fazer sua escolha profissional e de se preparar para os exames de admissão das universidades (GONZAGA; LIPP, 2014). Assim sendo, o Exame Nacional de Ensino Médio (ENEM), antigo exame vestibular, torna-se um forte gerador de estresse, que pode se manifestar através de tensões exacerbadas, problemas de memória, irritabilidade, sonolência, perda de concentração, entre outros sintomas (ARAÚJO et al., 2012; FERNANDES et al., 2017)

Alguns autores apontam a habilidade de lidar com o estresse como um importante elemento para o sucesso do jovem no ENEM (ARAÚJO et al., 2012 FARIA; WEBER; TON, 2012). Entretanto, nem todos os indivíduos estão preparados para lidar com o ritmo de estudos e simulados, cobranças dos pais, responsabilidades de sucesso, e acabam desencadeando sintomas de estresse físicos e/ou psicológicos (GONZAGA; LIPP, 2014; PEIXOTO et al., 2018).

Nesse sentido, dados nacionais demonstram que durante o período de preparação para o ENEM, em média, 62\% dos estudantes manifestam alguma forma de estresse, sendo mais prevalente no sexo feminino e com predominância dos sintomas psicológicos (FARIA; WEBER; TON, 2012; GONZAGA; LIPP, 2014; FERNANDES et al., 2017), que segundo Lipp, Malagris e Novais (2007), caracteriza-se por apatia, depressão, desânimo, ansiedade e irritabilidade. Percebe-se que todos esses fatores negativos podem comprometer o sucesso do estudante no ENEM.

O termo estresse, incorporado do inglês stress, é conceituado como uma reação do organismo frente a qualquer evento bom ou mau que altere a vida do indivíduo e que pode provocar o aparecimento de um conjunto de respostas orgânicas, psicológicas e/ou comportamentais (ARAÚJO et al., 2012; GONZAGA; LIPP, 2014). Nesse aspecto, percebe-se que o 


\section{CONGEESSO CIENCIAESOCIEDADE \\ Inovação, Diversidadie e Sustentahililitade}

estresse faz parte do dia a dia das pessoas; porém, quando se torna crônico, persistente e intenso isso pode causar sérios prejuízos à saúde (OLIVEIRA et al., 2019).

Pesquisas mostram que nas situações de estresse agudo o sistema neuroendócrino é ativado, desencadeando a liberação de vários hormônios, principalmente o cortisol, necessário às respostas orgânicas. Entretanto, quando o estresse se torna crônico há uma elevação na produção e liberação desse hormônio, causando diminuição da ação do sistema imunológico (MARSHALL et al., 2016; BAUER, 2019). Com seu sistema de defesa afetado, o organismo torna-se, então, mais vulnerável a infecções de vírus, bactérias e células cancerígenas. Em longo prazo, isso traz inúmeras implicações para a saúde pública, pois o indivíduo fica mais suscetível ao desenvolvimento de doenças crônicas não-transmissíveis, como hipertensão arterial, infartos agudos do miocárdio, acidentes vasculares cerebrais, câncer, úlceras, diabetes tipo II, síndrome metabólica, artrites, dores de cabeça crônicas, depressão e distúrbios nervosos (SOUSA et al., 2015; MCARDLE; KATCH; KATCH, 2016).

A literatura tem mostrado a eficácia de diferentes formas de tratamento para combater o estresse. O exercício físico tem feito parte dessas novas descobertas de tratamento, haja vista que vários autores têm afirmado que o exercício pode proteger o organismo dos efeitos prejudiciais do estresse (ARAÚJO et al., 2012; PEIXOTO et al., 2018; OLIVEIRA et al., 2019). Além disso, os exercícios físicos têm se mostrado eficazes na melhoria da memória e raciocínio, na qualidade do sono, no estado de humor, no aumento da disposição física e mental, dentre outros benefícios (FERRARI JÚNIOR et al., 2017; PINTO et al., 2018; PEREIRA et al., 2018).

No entanto, embora a prática de exercícios físicos seja benéfica à saúde, de modo geral, estudos epidemiológicos evidenciam a redução dos níveis de atividade física durante a adolescência à medida que os compromissos estudantis aumentam (ROLIM et al., 2010). Alguns estudos parecem corroborar com essa premissa, pois indicam elevada prevalência de sedentarismo entre os adolescentes brasileiros, variando de $22,2 \%$ no sexo masculino a $94 \%$ no sexo feminino em Niterói/RJ (TASSIANO et al., 2007; SILVA JÚNIOR et al., 2017).

Considerando os fatores apresentados, este estudo teve o propósito de investigar os níveis de atividade física (NAF) e os níveis de estresse de estudantes do ensino médio que estão se preparando para os exames de admissão das universidades, bem como identificar os sintomas e a fase do estresse em que esses estudantes se encontram, por meio de um indicador psicológico (escala de stress para adolescentes). 


\section{CONQGESSOC CIENCIAESOCIEDADE

\section{MATERIAL E MÉTODOS}

\section{Caracterização da Pesquisa}

A presente pesquisa consistiu em uma abordagem quantitativa, de natureza descritiva e de delineamento transversal. O projeto recebeu parecer de aprovação do Comitê de Ética em Pesquisa da Universidade Federal do Piauí/UFPI, protocolo 0311.0.045.000-10, e acompanhou as normas éticas da resolução CNS no 466/2012, do Conselho Nacional de Saúde sobre pesquisa, envolvendo seres humanos.

\section{Amostra}

A população desta pesquisa foi constituída de adolescentes, na faixa etária de 17 a 18 anos de idade, de ambos os sexos, estudantes do 3ㅇ (terceiro) ano do ensino médio de um colégio particular, da cidade de Teresina (PI). Para a realização do estudo, o colégio foi selecionado por conveniência.

Avaliou-se $n=141$ estudantes matriculados no 3 o ano do ensino médio, os quais foram convidados voluntariamente a responder à Escala de Stress para Adolescentes (ESA) e ao Questionário Internacional de Atividades Física (IPAQ-versão curta). Os adolescentes receberam e assinaram o TCLE e aqueles menores de idade levaram para casa para que um dos seus responsáveis assinasse, autorizando sua participação na pesquisa.

\section{Instrumentos e Procedimentos para Coleta de Dados}

Para mensurar e avaliar as variáveis de estudo, utilizou-se os seguintes instrumentos e procedimentos:

a) Escala de Stress para Adolescentes (ESA) - esse instrumento é composto por 44 itens e foi utilizado para: mensurar os níveis de estresse dos adolescentes de ambos os sexos, na faixa etária dos 17 aos 18 anos; identificar a sintomatologia mais frequente (psicológica, cognitiva, fisiológica e interpessoal) e a fase do estresse em que o estudante se encontrava (alerta, resistência, quase-exaustão e exaustão).

A análise dos dados da ESA e a avaliação da presença de estresse foram realizadas pela psicóloga (CRP 11â/PI 0385) com auxílio da pesquisadora, pois a ESA é um instrumento que somente pode ser manipulado por um profissional da psicologia credenciado.

b) Questionário Internacional de Atividade Física (IPAQ- versão curta) - para avaliar o nível de atividade física (NAF) dos estudantes, utilizou-se a versão curta em português do IPAQ 
(International Physical Activity Questionnaire), desenvolvido pela Organização Mundial de Saúde e validado para adolescentes de ambos os sexos e maiores de 14 anos (GUEDES e GUEDES, 2005). O IPAQ-versão curta é composto por oito questões, conforme Anexo B, referentes à prática de esportes e de lazer, atividades domésticas, ocupacionais e a forma de deslocamento ativo (caminhar ou pedalar) para a escola ou trabalho. Para este estudo, a classificação foi feita nomeando os indivíduos que não acumularam um mínimo de 300 minutos semanais de atividades físicas como insuficientemente ativos (PELEGRINI e PETROSKI, 2009).

\section{Análise Estatística}

Utilizou-se o programa estatístico SPSS for Windows versão 13.0 para análise dos dados intra e intergrupos. Foram empregados procedimentos estatísticos descritivos expressos por freqüência, percentual, média e desvio padrão. A associação entre as variáveis do NAF e de estresse da ESA foram analisadas por meio do Teste Qui-Quadrado. Neste estudo, o nível de significância foi estabelecido em $p<0,05$.

\section{RESULTADOS E DISCUSSÃO}

A prevalência de adolescentes insuficientemente ativos na população $(n=141)$ de estudantes do 3 ㅇ ano do ensino médio, de uma escola particular, da cidade de Teresina foi de $71,6 \%(n=101)$ e a prevalência de estresse foi de $70,2 \%(n=99)$. A amostra foi constituída por 67 adolescentes do sexo masculino e 74 do sexo feminino, na faixa etária de 17 a 18 anos de idade.

A elevada prevalência de adolescentes classificados como insuficientemente ativos neste estudo (FIGURA 1), pode estar associada à preparação para o ENEM, que exige horas a mais de estudo extraclasse. Vale ressaltar que a escola pesquisada não oferta a disciplina de educação física aos alunos do 3ㅇ ano do ensino médio, o que contribui para o reduzido nível de atividade física dos adolescentes. Corroborando com os resultados encontrados, Rolim et al. (2010) afirmam que à medida que os compromissos estudantis aumentam, há um decréscimo do nível habitual de atividades físicas. Entretanto, a prevalência estimada no presente estudo é preocupante, haja vista que o sedentarismo é um fator de risco para inúmeras doenças crônicas degenerativas e transtornos psicossomáticos, e tende a aumentar com a idade (PELEGRINI e PETROSKI, 2009; MARQUES; GASPAROTTO; COELHO, 2014; SILVA JÚNIOR, 2017). 


\section{CONQGESSOC CIENCIAESOCIEDADE

NÍVEL DE ATIVIDADE FÍSICA

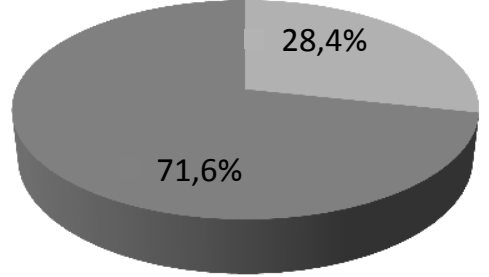

Ativos

Insuficientemente Ativos

Figura 1 Prevalência do nível de atividade física nos estudantes do 30 ano do ensino médio de uma escola particular de Teresina-PI.

Quanto à elevada prevalência de estresse nos vestibulandos (Figura 2), é provável que a explicação esteja associada a vários fatores relacionados à fase da adolescência, como: as inúmeras mudanças biopsicossociais que estão ocorrendo, os desequilíbrios hormonais, os conflitos psicossociais vivenciados, a necessidade da escolha profissional, a preparação para os exames vestibulares, e as pressões sociais para o êxito na admissão das universidades (FARIA; WEBER; TON, 2012; GONZAGA; LIPP, 2014; PEIXOTO et al., 2018).

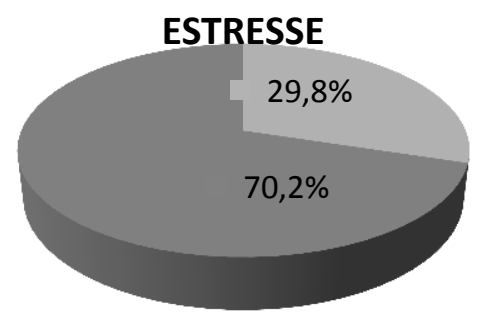

Ausente

Presente

Figura 2 Prevalência de nível de estresse nos estudantes do 3o ano do ensino médio de uma escola particular de Teresina-PI.

A elevada prevalência de estresse, encontrada neste estudo, corrobora com as evidências demonstradas na literatura de que o período de preparação para o ENEM é um forte agente estressor. No entanto, a grande preocupação é que o estresse crônico pode se manifestar em sensações somáticas como tensões exacerbadas, problemas de memória, dificuldade de raciocínio, irritabilidade, sonolência, apatia, perda de concentração, ansiedade, medo, insegurança, sentimentos de incompetência, aflição e até depressão (GONZAGA; LIPP, 2014; SILVA; LEONIDIO; FREITAS, 2015; PATIAS; HEINE; DELL'AGLIO, 2017). 


\section{CONGQEESSOCIENCIAESOCIEDADE

Segundo o modelo quadrifásico de Lipp et al. (2007), o estresse é composto pelas fases: alerta, resistência, quase-exaustão e exaustão. Conforme a Tabela 1, entre os adolescentes, a fase de resistência foi a predominante no sexo masculino $(\mathrm{SM})$, estando presente em $28,4 \%(\mathrm{n}=21)$ dos sujeitos e a fase de quase exaustão foi a mais prevalente no sexo feminino (SF), correspondendo a $23,9 \%(n=16)$ dos casos. Em seguida, encontrou-se a presença da fase de quase exaustão e de resistência em $27,0 \%(n=14)$ dos sujeitos do masculino e em $20,9 \%(n=14)$ das meninas, respectivamente. A fase mais branda do estresse (alerta) foi evidenciada em 17,6\% ( $n=13)$ no SM e em 14,9\% ( $n=10)$ no SF. Enquanto que a exaustão, a fase mais grave do estresse, foi verificada em $4 \%(n=3)$ dos participantes do SM e em $3 \%(n=2)$ do SF.

Tabela 1 Caracterização do estresse, quanto às fases e sintomas, nos vestibulandos de uma escola particular de Teresina-PI, 2011.

\begin{tabular}{|c|c|c|c|c|c|c|c|}
\hline \multirow[t]{2}{*}{ Variáveis } & \multicolumn{3}{|c|}{ Masculino $(n=74)$} & \multicolumn{3}{|c|}{ Feminino $(n=67)$} & \multirow[t]{2}{*}{ p-valor* } \\
\hline & $\mathbf{N}$ & $\%$ & $\begin{array}{c}\text { Intervalo de } \\
\text { Confiança de } 95 \% \\
\end{array}$ & $\mathbf{N}$ & $\%$ & $\begin{array}{c}\text { Intervalo de Confiança } \\
\text { de } 95 \%\end{array}$ & \\
\hline Fase & & & & & & & 0,460 \\
\hline Ausência & 17 & 23,0 & $(14,0 ; 34,2)$ & 25 & 37,3 & $(25,8 ; 50,0)$ & \\
\hline Alerta & 13 & 17,6 & $(9,7 ; 28,2)$ & 10 & 14,9 & $(7,4 ; 25,7)$ & \\
\hline Resistência & 21 & 28,4 & $(18,5 ; 40,1)$ & 14 & 20,9 & $(11,9 ; 32,6)$ & \\
\hline Quase exaustão & 20 & 27,0 & $(17,4 ; 38,6)$ & 16 & 23,9 & $(14,3 ; 35,6)$ & \\
\hline Exaustão & 03 & 4,0 & $(0,8 ; 11,4)$ & 02 & 3,0 & $(0,3 ; 10,4)$ & \\
\hline Sintomas & & & & & & & 0,416 \\
\hline Ausência & 17 & 23,0 & $(14,0 ; 34,2)$ & 25 & 37,3 & $(25,8 ; 50,0)$ & \\
\hline Psicológicos & 21 & 28,4 & $(18,5 ; 40,1)$ & 16 & 23,9 & $(14,3 ; 35,9)$ & \\
\hline Cognitivos & 12 & 16,2 & $(8,7 ; 26,6)$ & 09 & 13,4 & $(6,3 ; 24,0)$ & \\
\hline Fisiológicos & 13 & 17,6 & $(9,7 ; 28,2)$ & 11 & 16,4 & $(8,5 ; 27,5)$ & \\
\hline Interpessoal & 11 & 14,8 & $(7,7 ; 25,0)$ & 06 & 9,0 & $(3,4 ; 18,5)$ & \\
\hline
\end{tabular}

*Qui-quadrado de Person/Person's chi-square test

A literatura nacional que trata sobre a fase de estresse presente na população de adolescentes, estudantes do ensino médio, apresenta divergências dos resultados aqui encontrados para o SF, haja vista que o maior percentual das adolescentes se encontra na fase quase-exaustão; no entanto, os resultados do SM corroboram com as dados nacionais que indicam a fase de resistência (ARAÚJO et al., 2012; PEIXOTO et al., 2018; OLIVEIRA et al., 2019).

É necessário mencionar que a fase de quase exaustão caracteriza-se pelo desgaste físico e mental, pois a tensão excedeu a capacidade de adaptação do indivíduo ao estresse. A ansiedade eleva-se demasiadamente nessa fase, afetando o sistema imunológico que fica mais vulnerável e suscetível às infecções virais e bacterianas, além do desenvolvimento de doenças leves (LIPP, 


\section{cONGQESSO CIENCIASSOCIEDADE

MALAGRIS e NOVAIS, 2007). A memória e o raciocínio também ficam comprometidos, reduzindo a produtividade acadêmica (GONZAGA; LIPP, 2014). Caso não haja intervenção profissional e o estresse se prolongue ou se intensifique, o indivíduo pode entrar no próximo estágio que é a fase de exaustão. Nessa fase, o adolescente diminui seu ritmo de estudo, sua capacidade de concentração e pode desenvolver doenças graves como úlceras, hipertensão, diabetes, psoríase, vitiligo e depressão (LIPP, MALAGRIS e NOVAIS, 2007).

Como consequência desse desgaste orgânico do estudante em se adaptar às fases do estresse, vários sintomas foram observados e categorizados em quatro grupos, segundo a ESA: psicológico, cognitivo, fisiológico e interpessoal. A Figura 2 mostra a prevalência dos sintomas do estresse nos estudantes do SM e do SF.

Os resultados obtidos apontam que $28,4 \%(n=21)$ dos estudantes do SM apresentaram sintomatologia psicológica, seguido do sintoma fisiológico com $17,6 \%(n=13)$, do cognitivo com $16,2 \%(n=12)$ e do interpessoal com $14,8 \%(n=11)$. No SF, a sintomatologia predominante foi a psicológica, manifestada em $23,9 \%(n=16)$ das estudantes. Os demais sintomas do SF ficaram percentualmente assim distribuídos: fisiológico com $16,4 \%(n=11)$, cognitivo com 13,4\% ( $n=9)$, e interpessoal com $9 \%(n=6)$.

A literatura enfatiza que a sintomatologia de maior prevalência entre os estudantes que estão se preparando para os vestibulares é a psicológica. Nesse sentido, os achados da amostra atual convergem para os resultados evidenciados por Magalhães Neto e França (2003), em Brasília; Gonzaga e Lipp (2014), em São Paulo; Marques, Gasparotto e Coelho (2014), em revisão sistemática; Oliveira et al. (2019), em Teresina entre outros. No entanto, é importante ressaltar que todos estes estudos utilizaram o instrumento Inventário de Stress para Adultos de Lipp (ISSL), para avaliação do estresse dos adolescentes, e a presente pesquisa utilizou a Escala de Stress para Adolescentes (ESA). No ISSL, os sintomas são divididos em apenas duas categorias: psicológicos e físicos; e na ESA, os sintomas são categorizados em quatro grupos conforme figura 4, apresentada anteriormente. Deve-se considerar como ponto forte deste estudo o fato de ter sido utilizado um instrumento validado para avaliar o estresse do público adolescente.

De acordo com a escala de estresse, os sintomas psicológicos estão relacionados à tensão, agressividade, ansiedade, impaciência, emotividade excessiva, insegurança, apatia, irritabilidade, baixa autoestima, intolerância, tristeza, depressão, sensação de fadiga e ao desânimo (TRICOLI e LIPP, 2005). 


\section{CONGEESSO CIENCIAESOCIEDADE Inovação, Diversidadie e Sustentahililitade}

Nesse sentido, a literatura científica tem sugerido o exercício físico como uma das principais medidas para o alívio do estresse. No entanto, são escassas as pesquisas nacionais que utilizam o exercício físico como tratamento não farmacológico para o público adolescente estressado que está na fase de preparação para o ENEM, haja vista que o processo seletivo das universidades em outros países difere do ocorrido no Brasil.

Tendo em vista esse contexto, buscou-se investigar a associação entre a prevalência de estresse e o nível de atividade física (NAF) dos estudantes. Conforme Tabela 2, houve associação significativa entre a presença de estresse avaliada pela ESA e o fato de o estudante estar insuficientemente ativo $(p<0,004)$. Portanto, nesta amostra, o fato de o adolescente se perceber estressado pela escala $(77,2 \%)$, teve associação significativamente positiva com o baixo nível de atividades físicas diárias, de acordo com os resultados do teste Qui-quadrado.

Tabela 2 Associação entre a prevalência de estresse e o nível de atividade física (NAF) em estudantes do 3 ㅇ ano do ensino médio de uma escola particular de Teresina-PI, 2011.

\begin{tabular}{|c|c|c|c|c|c|}
\hline \multirow[t]{4}{*}{ Variáveis } & \multicolumn{4}{|c|}{ NAF } & \multirow{4}{*}{ p-valor* } \\
\hline & \multirow{2}{*}{\multicolumn{2}{|c|}{$\begin{array}{l}\text { Ativos } \\
(n=40)\end{array}$}} & \multirow{2}{*}{\multicolumn{2}{|c|}{$\begin{array}{l}\text { Insuficientemente } \\
\text { Ativos ( } n=101)\end{array}$}} & \\
\hline & & & & & \\
\hline & $\mathbf{N}$ & $\%$ & $\mathbf{N}$ & $\%$ & \\
\hline \multicolumn{6}{|l|}{ Estresse } \\
\hline Ausência & 19 & 47,5 & 23 & 22,8 & \multirow[t]{2}{*}{0,004} \\
\hline Presença & 21 & 52,5 & 78 & $77,2^{*}$ & \\
\hline
\end{tabular}

*Qui-quadrado de Person/Person's chi-square test

Os dados sugerem que a maior vulnerabilidade ao estresse, desses estudantes da amostra, pode estar associada ao fato dos participantes se encontrarem insuficientemente ativos. Nesse particular, Peixoto et al. (2018) evidenciaram, em seu estudo, que quanto maior o sedentarismo maior era o nível de estresse prejudicial à saúde.

Nessa linha de pensamento, Araújo et al. (2012) e Silva, Leonidio e Freitas (2015) asseveram que o sedentarismo pode potencializar os efeitos nocivos do estresse, pois o indivíduo insuficientemente ativo tende a ter maiores sobrecargas metabólicas imposta aos seus sistemas: cardiovascular, imune e muscular. Desse modo, os sintomas físicos e psicológicos devem ser diagnosticados e tratados, caso contrário, podem se somatizar em doenças mais severas (GONZAGA; LIPP, 2014; PATIAS; HEINE; DELL'AGLIO, 2017; BAUER, 2019). 


\section{CONSIDERAÇÕES FINAIS}

Nesse sentido, a prática regular de atividades físicas poderia contribuir para evitar a elevada prevalência de adolescentes insuficientemente ativos do ensino médio, presente nesta pesquisa; haja vista que o sedentarismo é um dos principais fatores de risco para o desenvolvimento de doenças crônico-degenerativas e de transtornos psicossomáticos como, por exemplo, o estresse. Tal fato parece ser verdadeiro, pois a maioria dos estudantes apresentou estar estressada.

Com relação às fases e sintomas do estresse predominantes, verificou-se que a maioria dos estudantes do SM encontrava-se na fase de resistência, enquanto que o SF encontrava-se na fase de quase exaustão. A amostra apresentou predominância dos sintomas psicológicos.

Quanto à associação entre o NAF e os níveis de estresse percebido (ESA), as informações estatísticas selecionadas no estudo indicam que houve uma relação significativa entre o sedentarismo e o estresse, sugerindo que a aproximação da data dos exames nacionais contribuiu para aumentar os sintomas nessa amostra de estudantes.

Diante dos resultados encontrados, pode-se inferir que o sedentarismo pode potencializar os efeitos nocivos do estresse, durante essa etapa da vida. No entanto, sugere-se a realização de novas pesquisas, com delineamento semelhante a esta, porém envolvendo amostras maiores e com estudantes de escolas particulares e públicas, para verificar se os achados serão semelhantes aos encontrados neste estudo.

\section{REFERÊNCIAS}

ARAÚJO, M. C. et al. Efeitos do exercício físico sobre os níveis de estresse em vestibulando de Teresina-PI. Revista Brasileira de Ciência e Movimento, v. 20, n. 3, p. 14-26, 2012.

FARIA, R. R.; WEBER, L. N. D.; TON, C. T. O estresse entre vestibulandos e suas relações com a família e a escolha profissional. Psicologia Argumento, v. 30, n.68, p. 43-52, 2012.

FERNANDES, R. L. et al. Relação entre estresse, atividade física e desempenho escolar em adolescentes. Arquivo de Ciências do Esporte, v.5, n.2, p.37-39, 2017.

FERRARI JÚNIOR, G. J. et al. A baixa duração do sono está associada com a inatividade física em adolescentes amazonenses. Revista Brasileira de Atividade Física e Saúde, v.22, n.4, p. 373-381, 2017. 
GONZAGA, L. R. V.; LIPP, M. E. Relação entre escolha profissional, vocação e nível de estresse em estudantes do ensino médio. Psicologia Argumento, v. 32, n.78, p. 149-156, 2014.

GUEDES, D.P.; LOPES, C.C.; GUEDES, J.E. Reprodutibilidade e validade do Questionário Internacional de Atividade Física em adolescentes. Revista Brasileira de Medicina do Esporte, v. 11, p. 151-158, 2005.

LIPP, M. E. N.; MALAGRIS, L. E. N.; NOVAIS, L. E. Stress ao longo da vida. São Paulo: Ícone, 2007.

MARQUES, C. P.; GASPAROTTO, G. S.; COELHO, R. W. Fatores relacionados ao nível de estresse em adolescentes estudantes: uma revisão sistemática. Salusvita , v. 34, n.1, p. 99-108, 2015.

MARSHALL, W. J.; LAPSLEY, M; DAY, A. P.; AYLIN, R. M. Bioquímica Clínica: Aspectos Clínicos e Metabólicos. 3a ed. Rio de Janeiro: Elsevier, 2016. 976 p.

McARDLE, W. D.; KATCH, F. I.; KATCH, V. L. Fisiologia do Exercício - Nutrição, Energia e Desempenho Humano. 8a ed. Guanabara Koogan, 2016. 1120 p.

NETO MAGALHÃES, A. M; FRANÇA, N. M. Efeitos de um programa de exercícios resistidos sobre o estresse mental em estudantes do Ensino Médio. Revista Brasileira Ciência e Movimento, Brasília, v. 11, n. 4, p. 33-36, 2003.

OLIVEIRA, V. A. S. et al. Associação entre o nível de atividade física e o estresse em prévestibulando. Revista Brasileira de Fisiologia do Exercício, v. 18, n. 1, p. 9-16, 2019.

PATIAS, N. D.; HEINE, J. A.; DELL'AGLIO, D. D. Bem-estar subjetivo, violência e sintomas de depressão, ansiedade e estresse em adolescentes. Avaliação Psicológica, v.16, n.4, p.468-477, 2017.

PEIXOTO, M. B. et al. Atividade física e estresse psicológico em adolescentes: uma revisão sistemática. Saúde em Redes, v., n.1, p. 14-159, 2018.

PELEGRINI, Andreia; PETROSKI, Edio Luiz. Inatividade física e sua associação com estado nutricional, insatisfação com a imagem corporal e comportamentos sedentários em adolescentes de escolas públicas. Revista Paulista de Pediatria, v.27, n.4, p. 366-373, 2009.

PEREIRA, G. P. et al. Insônia: o benefício do exercício ísico em adolescentes com excesso de peso. Journal Health Biology Science, v.6, n.4, p.377-382, 2018.

PINTO, A. A. et al. Qualidade do sono de adolescentes que trabalham e não praticam atividade física. Caderno Brasileiro de Terapia Ocupacional, v.6 , n.1, p.137-143, 2018.

ROLIM, M. K. S. B.; MATIAS, T. S.; CUNHA, M. L. da; ANDRADE, A. Estilo de vida de adolescentes ativos e sedentários. Trabalho apresentado no: 6 ENCONTRO INTERNACIONAL DE ESPORTES, 


\section{cONQ⿻口卄ESSO CIENCIASSOCIEDADE \\ Inovação, Diversidaale e Sustentahilitilade}

2007,

Florianópolis.

Disponível

em:

http://www.unesporte.org.br/forum2007/apresentacao.html>. Acesso em: 18 jul. 2019.

SOUSA, M. B. C. et al. Resposta ao estresse: I. Homeostase e teoria da alostase. Estudos de Psicologia, v.20, n.1, p.2-11, 2015.

SILVA, M. L.; LEONIDIO, A. C. R.; FREITAS, C. M. S. M. Atividade física e o estresse psicossocial frente ao adolescer a luz da sociedade moderna. Revista Brasileira de Ciência e Movimento, v. 23, n. 23, p. 170-178, 2015.

SILVA JÚNIOR, F. G. et al. Sedentarismo e inatividade física em adolescentes com faixa etária de escolares do ensino médio e reflexões para educação física escolar. Revista Saúde Física e Mental, v. 5, n. 1, 2017.

TASSITANO, R. M.; BEZERRA, J.; TENÓRIO, M.C.; COLARES, V.; BARROS, M. V.; HALLAL, P. C. Atividade física em adolescentes brasileiros: uma revisão sistemática. Revista Brasileira de Cineantropometria e Desempenho Humano, v. 9, p.55-60, 2007.

TRICOLI, Valquiria Aparecida Cintra; LIPP, Marilda Emmanuel Novaes. ESA: Escala de stress para adolescentes. São Paulo: Casa do Psicólogo, 2005. 